\title{
The preventive effect of $\beta$-carotene on denervation-induced soleus muscle atrophy in mice
}

\author{
Masahiro Ogawa $^{1}$, Yoshihiro Kariya ${ }^{1}$, Tomoya Kitakaze ${ }^{1}$, Ryoichi Yamaji ${ }^{1 *}$, Naoki Harada ${ }^{1}$, \\ Tatsuji Sakamoto $^{1}$, Keisuke Hosotani ${ }^{2}$, Yoshihisa Nakano ${ }^{3}$ and Hiroshi Inui ${ }^{1}$ \\ ${ }^{1}$ Division of Applied Life Sciences, Graduate School of Life and Environmental Sciences, Osaka Prefecture University, \\ 1-1 Gakuen-cho, Naka-ku, Sakai, Osaka 5998531, Japan \\ ${ }^{2}$ Faculty of Life Welfare, Seibi University Junior College, 3370 Aza Hori, Fukuchiyama, Kyoto 6200886, Japan \\ ${ }^{3}$ Department of Life Sciences, Osaka Women's Junior College, 3-8-1 Kasugaoka, Fujiidera, Osaka 5838558, Japan
}

(Submitted 27 January 2012 - Final revision received 15 June 2012 - Accepted 25 June 2012 - First published online 9 October 2012)

\section{Abstract}

Muscle atrophy increases the production of reactive oxygen species and the expression of atrophy-related genes, which are involved in the ubiquitin-proteasome system. In the present study, we investigated the effects of $\beta$-carotene on oxidative stress $\left(100 \mu \mathrm{m}-\mathrm{H}_{2} \mathrm{O}_{2}\right)$-induced muscle atrophy in murine $\mathrm{C} 2 \mathrm{C} 12$ myotubes. $\beta$-Carotene $(10 \mu \mathrm{M})$ restored the $\mathrm{H}_{2} \mathrm{O}_{2}$-induced decreased levels of myosin heavy chain and tropomyosin $(P<0 \cdot 05, n 3)$ and decreased the $\mathrm{H}_{2} \mathrm{O}_{2}$-induced increased levels of ubiquitin conjugates. $\beta$-Carotene reduced the $\mathrm{H}_{2} \mathrm{O}_{2-}$ induced increased expression levels of E3 ubiquitin ligases (Atrogin-1 and MuRF1) and deubiquitinating enzymes (USP14 and USP19) $(P<0 \cdot 05, n 3)$ and attenuated the $\mathrm{H}_{2} \mathrm{O}_{2}$-induced nuclear localisation of FOXO3a. Furthermore, we determined the effects of $\beta$-carotene on denervation-induced muscle atrophy. Male ddY mice ( 8 weeks old, $n$ 30) were divided into two groups and orally pre-administered micelle with or without $\beta$-carotene $(0.5 \mathrm{mg}$ once daily) for 2 weeks, followed by denervation in the right hindlimb. $\beta$-Carotene was further administered once daily until the end of the experiment. At day 3 after denervation, the ratio of soleus muscle mass in the denervated leg to that in the sham leg was significantly higher in $\beta$-carotene-administered mice than in control vehicle-administered ones $(P<0 \cdot 05, n 5)$. In the denervated soleus muscle, $\beta$-carotene administration significantly decreased the expression levels of Atrogin-1, MuRF1, USP14 and USP19 $(P<0 \cdot 05, n 5)$ and the levels of ubiquitin conjugates. These results indicate that $\beta$-carotene attenuates soleus muscle loss, perhaps by repressing the expressions of Atrogin-1, MuRF1, USP14 and USP19, at the early stage of soleus muscle atrophy.

Key words: $\beta$-Carotene: Muscle atrophy: Ubiquitin ligase: Deubiquitinating enzymes: Denervation

Skeletal muscle is the most abundant tissue in the human body. The functions of skeletal muscle are glucose and lipid metabolism in addition to mobility and movement. A prolonged period of skeletal muscle disuse due to denervation, hindlimb unloading, immobilisation or bed rest leads to muscle atrophy, thereby decreasing the mass and function of skeletal muscle ${ }^{(1)}$. A potential factor in the loss of muscle mass is oxidative stress. The production of reactive oxygen species increases in muscle mitochondria during denervation, hindlimb unloading, and sarcopenia ${ }^{(2,3)}$. Antioxidants are expected to attenuate the muscle disuse-induced increase in reactive oxygen species emission and to prevent skeletal muscle from oxidative stress ${ }^{(1,4,5)}$. However, such antioxidants have not yet been identified.

The balance between the synthesis and degradation of muscle proteins such as myosin heavy chain (MyHC) and tropomyosin regulates muscle mass, and increased protein degradation results in a loss of muscle mass. Muscle atrophy increases the expression of atrophy-related genes (atrogenes) such as muscle-specific E3 ubiquitin ligases (Atrogin-1 and MuRF1) ${ }^{(6-9)}$. Okamoto et al. ${ }^{(7)}$ and Sacheck et al. ${ }^{(8)}$ reported that the expressions of Atrogin-1 and MuRF1 are dramatically increased during the early stage or early phase (e.g. at $3 \mathrm{~d}$ ) of disuse atrophy induced by immobilisation and denervation, respectively. The ubiquitin-proteasome system controls the degradation of aberrant proteins. Atrogin-1 and MuRF1 conjugates ubiquitin to muscle proteins, and subsequently the conjugated proteins are degraded by the proteasome. Because Atrogin-1 and MURF1 knockout mice are resistant to muscle atrophy $^{(6)}$, the down-regulation of Atrogin-1 and MuRF1 expressions should contribute to the prevention of muscle atrophy. Many atrogenes are induced by the FOXO family of

Abbreviations: DMEM, Dulbecco's modified Eagle's medium; DUB, deubiquitinating enzyme; GAPDH, glyceraldehyde-3-phosphate dehydrogenase; MyHC, myosin heavy chain; TBARS, thiobarbituric acid-reactive substances; USP, ubiquitin-specific peptidase.

*Corresponding author: Professor R. Yamaji, fax +81 72254 9921, email yamaji@biochem.osakafu-u.ac.jp 
transcription factors ${ }^{(10)}$. Oxidative stress is involved in the activation of FOXO3a, and activated FOXO3a induces the expression of Atrogin-1 and MuRF1 ${ }^{(10-13)}$. On the other hand, the ubiquitin-proteasome system is also regulated by the deubiquitination reaction, in which deubiquitinating enzymes (DUB) cleave ubiquitin from ubiquitin-conjugated proteins and recycle free ubiquitin ${ }^{(14)}$. DUB are called ubiquitin-specific peptidases (USP). Muscle atrophy increases the expression of USP14 and USP19 mRNA, and knockdown of USP19 inhibits muscle atrophy ${ }^{(15-17)}$. Therefore, the expression levels of ubiquitin ligases and DUB should be monitored during muscle atrophy.

Edible plant-derived antioxidants such as carotenoids provide some protection against oxidative stress. $\beta$-Carotene is a dietary source of vitamin $\mathrm{A}^{(18)}$ and has been shown to have beneficial effects in vitro as an antioxidant ${ }^{(19-22)}$. However, it remains unclear whether dietary supplementation of $\beta$-carotene attenuates disuse-induced muscle atrophy. Here, we report that oxidative stress increases USP14 and USP19 expressions in addition to Atrogin-1 and MuRF1 and that $\beta$-carotene inhibits their increased gene expression in vitro. Furthermore, we demonstrate that oral administration of $\beta$-carotene to mice attenuates denervation-induced loss of soleus muscle mass and inhibits increases in the expressions of two ubiquitin ligases and two DUB at the early stage of disuse atrophy.

\section{Experimental methods}

\section{Cell culture}

Murine skeletal muscle $\mathrm{C} 2 \mathrm{C} 12$ myoblasts were obtained from the RIKEN Cell Bank (Ibaraki, Japan). C2C12 myoblasts were cultured in Dulbecco's modified Eagle's medium (DMEM) supplemented with 10\% fetal bovine serum, 100 units penicillin/ml, and $100 \mu \mathrm{g}$ streptomycin/ml (growth medium) at $37^{\circ} \mathrm{C}$ in a $5 \% \mathrm{CO}_{2}-95 \%$ air atmosphere at $100 \%$ humidity. To induce differentiation from myoblasts to myotubes, C2C12 myoblasts were grown to $90 \%$ confluency in the growth medium and shifted to DMEM supplemented with $2 \%$ horse serum (differentiation medium). The differentiation medium was replaced at $48 \mathrm{~h}$ intervals for $8 \mathrm{~d}$. When cells were incubated in the presence of $\beta$-carotene, $\beta$-carotene was dissolved in dimethyl sulfoxide. As a vehicle control, dimethyl sulfoxide was added at a final concentration of $0 \cdot 1 \%$.

\section{Animals and $\beta$-carotene administration}

The care of all animals in the present study was in accordance with the guidelines of the Animal Care and Use Committee of Osaka Prefecture University. A total of thirty male Kwl:ddY mice ( 7 weeks old) were obtained from Kiwa Laboratory Animals (Wakayama, Japan). These mice had free access to water and a non-purified diet (CE-2; Clea Japan, Inc.) and were kept at a controlled temperature $\left(23 \pm 2^{\circ} \mathrm{C}\right)$, humidity $(60 \pm 10 \%)$ and lighting (a $12 \mathrm{~h}$ light$12 \mathrm{~h}$ dark cycle starting at 08.00 hours). After 1 week of adaptation ( 8 weeks old), mice (30-35 g body weight) were randomly divided into two groups ( $n 15$ per group). One group was orally administered micellar $\beta$-carotene $(0.5 \mathrm{mg}$ once daily) by direct intubation to the stomach ( $\beta$-carotene group) and the other was orally administered micelles without $\beta$-carotene as a vehicle (vehicle group) for 2 weeks. Micellar $\beta$-carotene was prepared as mixed micelles as described previously $^{(23)}$. In brief, $\beta$-carotene $(5 \mathrm{mg})$ and sodium taurocholate $(12.9 \mathrm{mg})$ were mixed in ethanol $(0.5 \mathrm{ml})$ and dried under $\mathrm{N}_{2}$ gas. The residue was mixed in lysophosphatidyl choline $(1.31 \mathrm{mg})$, oleic acid $(4.23 \mathrm{mg})$ and mono-olein $(1.78 \mathrm{mg})$ in distilled water $(2 \mathrm{ml})$ under shading.

\section{Denervation of hindlimb and tissue harvest}

A 4 to $5 \mathrm{~mm}$ segment of the sciatic nerve in the right hindlimb of 10-week-old mice ( $n 10$ per group) was removed under pentobarbital $(50 \mathrm{mg} / \mathrm{kg}$ body weight, intraperitoneal) anaesthesia, and the contralateral non-denervated left hindlimb was sham-operated as an internal control. $\beta$-Carotene was further administered $(0.5 \mathrm{mg}$ once daily) until the end of the experiment. Food intake in the experiment was controlled because the non-denervated hindlimb from the same animal was used as a control. The animals in each group were killed at 3 and $7 \mathrm{~d}$ ( $n 5$ per time point) after denervation under pentobarbital $(50 \mathrm{mg} / \mathrm{kg}$ body weight intraperitoneal) anaesthesia (days 3 and 7 , respectively). On the other hand, ten 10-week-old mice ( $n 5$ per group) were killed without denervation (day 0). The skeletal muscle tissues (soleus and gastrocnemius muscles) were excised, cleaned off extraneous tissues and weighed. The sample was frozen in liquid $\mathrm{N}_{2}$ and stored at $-80^{\circ} \mathrm{C}$ until use.

\section{RNA isolation and RT-PCR analysis}

C2C12 myotubes were pre-incubated in the presence of vehicle or $\beta$-carotene for $12 \mathrm{~h}$, followed by rinsing twice with PBS, $\mathrm{pH} 7 \cdot 4$, and incubation in fresh DMEM differentiation medium containing $100 \mu \mathrm{M}-\mathrm{H}_{2} \mathrm{O}_{2}$ for $3 \mathrm{~h}$. Total RNA was isolated using Sepasol-RNA II Super G (Nacalai Tesque) according to the manufacturer's instructions, and reverse-transcribed. On the other hand, total RNA from skeletal muscle was extracted from frozen tissues. The resultant complementary DNA were subjected to quantitative real-time PCR using the following specific primers: Atrogin-1 (forward primer 5'TCACAGCTCACATCCCTGAG-3' and reverse primer $5^{\prime}$-GACTTGCCGACTCTCTGGAC-3'); MuRF1 (forward primer 5'-GACTCCTGCAGAGTGACCAAG- $3^{\prime}$ and reverse primer $5^{\prime}$-CTTCTACAATGCTCTTGATGAGC-3'); USP14 (forward primer 5'-CCAAGCCACTTGTGCATCTTCA- $3^{\prime}$ and reverse primer $5^{\prime}$-TAGATAAGGCAGAGGACGGGCA- $3^{\prime}$ ). Specific primers for USP19, glyceraldehyde-3-phosphate dehydrogenase $(G A P D H)$ and $\beta$-actin were as described previously ${ }^{(24)}$. The PCR profiles consisted of denaturation at $95^{\circ} \mathrm{C}$ for $1 \mathrm{~min}$, primer annealing at $57^{\circ} \mathrm{C}$ for $1 \mathrm{~min}$ and primer extension at $72^{\circ} \mathrm{C}$ for $30 \mathrm{~s}$. The final primer extension was performed at $72^{\circ} \mathrm{C}$ for $10 \mathrm{~min}$. The PCR in quantitative real-time PCR was performed with the Plexor One-Step qRT-PCR System (Promega) on a Thermal Cycler Dice, TP-800 (Takara). $C_{\mathrm{t}}$ values were transformed into 
relative quantification data by the $2^{-\Delta \Delta C_{t}}$ method, and were normalised to the $C_{\mathrm{t}}$ values of $\beta$-actin or GAPDH.

\section{Western blotting}

C2C12 myotubes were pre-incubated in the presence of vehicle or $\beta$-carotene for $12 \mathrm{~h}$ and rinsed twice with PBS, followed by incubation in fresh DMEM differentiation medium containing $100 \mu \mathrm{M}-\mathrm{H}_{2} \mathrm{O}_{2}$. To accumulate ubiquitin-conjugated proteins, myotubes were incubated in the presence of $10 \mu \mathrm{M}-\mathrm{MG} 132$. After $12 \mathrm{~h}, \mathrm{C} 2 \mathrm{C} 12$ myotubes were lysed in lysis buffer A (50 mm-Tris- $\mathrm{HCl}, \mathrm{pH} 7 \cdot 5$, containing $150 \mathrm{~mm}-\mathrm{NaCl}$, $0 \cdot 25 \%$ sodium deoxycholate, $1 \%$ Nonidet P-40, 0.1\% SDS, 1 mm-EDTA, 1 mm-4-(2-aminoethyl)benzenesulfonyl fluoride, $10 \mu \mathrm{g}$ leupeptin/ml and $1 \mu \mathrm{g}$ aprotinin/ml) and sonicated. On the other hand, soleus and gastrocnemius muscles $(5 \mathrm{mg})$ were homogenised in lysis buffer B (20 mM-HEPES$\mathrm{NaOH}, \mathrm{pH} 7 \cdot 5$, containing $0 \cdot 5 \%$ Nonidet P-40, 1 mm-EDTA, $50 \mu \mathrm{m}$-butylated hydroxytoluene, $1 \mathrm{~mm}$-4-(2-aminoethyl) benzenesulfonyl fluoride, $10 \mu \mathrm{g}$ leupeptin/ml and $1 \mu \mathrm{g}$ aproti$\mathrm{nin} / \mathrm{ml}$ ). Lysates were subjected to SDS-PAGE and analysed by Western blotting with the following primary antibodies: rabbit polyclonal anti-GAPDH $(1: 5000)^{(25)}$, and mouse monoclonal anti-MyHC (1:2000) (MF-20, supernatant; Developmental Studies Hybridoma Bank), anti-tropomyosin (1:2000) (CH1, supernatant; Developmental Studies Hybridoma Bank), and anti-ubiquitin antibodies (1:5000) (FK2; Nippon Biotest Laboratories, Inc.). Immunoreactive proteins were incubated with horseradish peroxidase-conjugated goat anti-rabbit $\operatorname{IgG}$ (1:3000) or anti-mouse $\operatorname{IgG}(1: 3000)$ and reacted with the Super Signal West Femto Chemiluminescent Substrate (Pierce Biotechnology) or Immobilon Western Chemiluminescent HRP Substrate (Millipore), followed by detection with the LAS4000 imaging system (GE Healthcare). The intensities of immunoreactive proteins were quantified by densitometry using Image J (version 1.44; National Institutes of Health).

\section{Immunofluorescence analysis}

C2C12 myotubes were pre-incubated in the presence of vehicle or $\beta$-carotene for $12 \mathrm{~h}$ and rinsed twice with PBS, followed by incubation in fresh DMEM differentiation medium containing $100 \mu \mathrm{M}-\mathrm{H}_{2} \mathrm{O}_{2}$ for $3 \mathrm{~h}$. C2C12 myotubes were fixed in $4 \%$ paraformaldehyde in PBS for $10 \mathrm{~min}$ and permeabilised with $0 \cdot 2 \%$ Triton $\mathrm{X}-100$ in PBS for $5 \mathrm{~min}$ as described previously $^{(26)}$. The cells were incubated with rabbit monoclonal anti-FOXO3a antibody (1:500) (75D8; Cell Signaling Technology) at $4^{\circ} \mathrm{C}$ overnight, followed by incubation with antirabbit Alexa 488-conjugated antibody (1:2000) (Cell Signaling Technology) at room temperature under shading. After $1 \mathrm{~h}$, the cells were counterstained with 4',6-diamidino-2-phenylindole dihydrochloride $(1 \mu \mathrm{g} / \mathrm{ml})$ in PBS for $10 \mathrm{~min}$. Negative controls were treated with only a secondary antibody. Fluorescent images were analysed by using a BIOREVO BZ-9000 fluorescence microscope (Keyence).
(A)

\begin{tabular}{|c|c|c|c|c|c|}
\hline $100 \mu \mathrm{M}-\mathrm{H}_{2} \mathrm{O}_{2}$ & - & + & + & + & + \\
\hline$\beta$-Carotene $(\mu \mathrm{M})$ & 0 & 0 & $0 \cdot 1$ & 1 & 10 \\
\hline $\mathrm{MyHC}$ & - & & & - & - \\
\hline Tropomyosin & - & - & $=$ & - & - \\
\hline
\end{tabular}

(B)

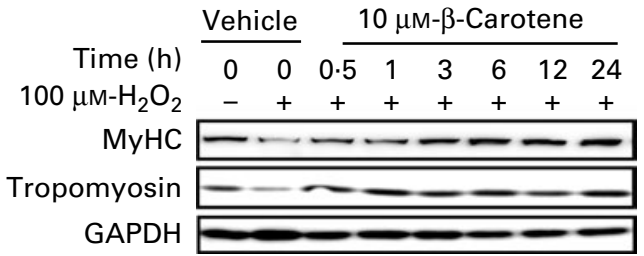

(C)
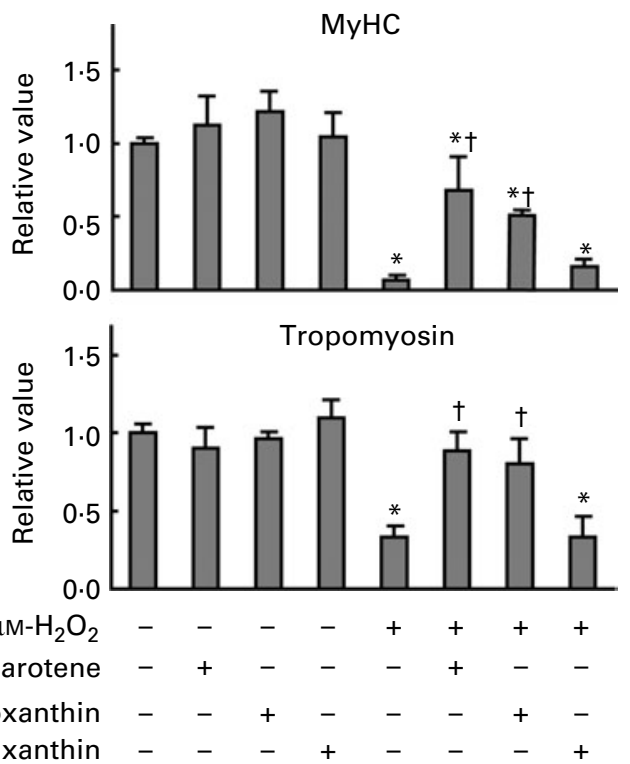

Fig. 1. Inhibitory effect of $\beta$-carotene on hydrogen peroxide-induced $\mathrm{C} 2 \mathrm{C} 12$ myotube atrophy. (A) $\mathrm{C} 2 \mathrm{C} 12$ myotubes were pre-incubated in the presence of various concentrations $(0-10 \mu \mathrm{M})$ of $\beta$-carotene for $12 \mathrm{~h}$, and further incubated in fresh medium containing $100 \mu \mathrm{M}$-hydrogen peroxide for $12 \mathrm{~h}$. Western blot analyses with anti-myosin heavy chain (MyHC), anti-tropomyosin and anti-glyceraldehyde-3-phosphate dehydrogenase (GAPDH) antibodies are shown. (B) C2C12 myotubes were pre-incubated in the presence of vehicle or $10 \mu \mathrm{M}-\beta$-carotene for various time periods, and further incubated in fresh medium containing $100 \mu \mathrm{M}$-hydrogen peroxide for $12 \mathrm{~h}$. Western blot analyses with anti-MyHC, anti-tropomyosin and anti-GAPDH antibodies are shown. (C) C2C12 myotubes were pre-incubated in the presence of vehicle or $10 \mu \mathrm{M}$-carotenoid ( $\beta$-carotene, $\beta$-cryptoxanthin or zeaxanthin) for $12 \mathrm{~h}$, and further incubated in fresh medium containing $100 \mu \mathrm{M}$-hydrogen peroxide for $12 \mathrm{~h}$. Western blot analyses with anti-MyHC, anti-tropomyosin and anti-GAPDH antibodies were performed, and the intensities of immunoreactive bands were quantified $(n 3)$. The levels of MyHC and tropomyosin were normalised to the level of GAPDH, and data were expressed as a relative value of the intensity of the control (hydrogen peroxide-untreated myotubes in the presence of vehicle). Values are means, with their standard deviations represented by vertical bars. Mean value was significantly different compared with hydrogen peroxide-untreated myotubes under the same carotenoid treatment conditions: ${ }^{*} P<0.05$. Mean value was significantly different compared with vehicle-treated myotubes under hydrogen peroxide treatment conditions: $† P<0.05$. 


\section{Thiobarbituric acid-reactive substances assay}

Lipid peroxidation was determined by measuring thiobarbituric acid-reactive substances (TBARS) according to the method of Ohkawa et $a l .{ }^{(27)}$. Soleus and gastrocnemius muscles were homogenised in lysis buffer B (20 mm-HEPES-NaOH, pH 7.5, containing $0.5 \%$ Nonidet P-40, 1 mm-EDTA, $50 \mu \mathrm{m}$-butylated hydroxytoluene, $1 \mathrm{~mm}$-4-(2-aminoethyl)benzenesulfonyl fluoride, $10 \mu \mathrm{g}$ leupeptin $/ \mathrm{ml}$ and $1 \mu \mathrm{g}$ aprotinin $/ \mathrm{ml}$ ), and the homogenates were subjected to the TBARS assay. The homogenates were incubated with $0.8 \%$ 2-thiobarbituric acid for $45 \mathrm{~min}$ at $90^{\circ} \mathrm{C}$. The organic layer was extracted with $n$-butanol, and fluorescence was measured at an excitation of $544 \mathrm{~nm}$ and an emission of $590 \mathrm{~nm}$ (Fluoroskan Ascent FL; Labsystems). The values are expressed in nmol malondialdehyde/mg protein.

\section{Statistics}

Data for RT-PCR analysis in C2C12 myotubes were analysed by one-way ANOVA with Tukey's post hoc test, and the others were analysed by two-way ANOVA with Bonferroni's post hoc test using GraphPad Prism 4 (GraphPad Software). Data are expressed as means and standard deviations, and differences were considered as statistically significant at $P<0.05$.

\section{Results}

\section{$\beta$-Carotene inhibits hydrogen peroxide-induced muscle} atrophy in C2C12 myotubes

We determined the effects of $\beta$-carotene on muscle atrophy induced by oxidative stress in $\mathrm{C} 2 \mathrm{C} 12$ myotubes. $\beta$-Carotene dose-dependently attenuated $\mathrm{H}_{2} \mathrm{O}_{2}$-induced decreases in MyHC and tropomyosin expression (Fig. 1(A)). When C2C12 myotubes were pre-incubated in the presence of $\beta$-carotene for various time periods $(0.5-24 \mathrm{~h})$, followed by treatment with $\mathrm{H}_{2} \mathrm{O}_{2}, \beta$-carotene time-dependently abolished $\mathrm{H}_{2} \mathrm{O}_{2}$ induced decreases in MyHC and tropomyosin expression (Fig. 1(B)). Furthermore, the effects of other carotenoids on $\mathrm{H}_{2} \mathrm{O}_{2}$-induced muscle atrophy were determined in $\mathrm{C} 2 \mathrm{C} 12$ cells. As with $\beta$-carotene, $\beta$-cryptoxanthin and zeaxanthin inhibited $\mathrm{H}_{2} \mathrm{O}_{2}$-induced decreases in MyHC and tropomyosin expression in the order $\beta$-carotene $=\beta$-cryptoxanthin $>$ zeaxanthin (Fig. 1(C)).

$\beta$-Carotene represses hydrogen peroxide-induced levels of ubiquitin ligases and deubiquitinating enzymes in C2C12 myotubes

$\beta$-Carotene repressed the levels of ubiquitin conjugates in a dose-dependent manner in $\mathrm{H}_{2} \mathrm{O}_{2}$-treated myotubes (Fig. 2). $\beta$-Carotene dose-dependently inhibited the $\mathrm{H}_{2} \mathrm{O}_{2}$-induced increased expressions of the two ubiquitin ligases (Fig. 3(A) and (B)). When $\mathrm{C} 2 \mathrm{C} 12$ myotubes were exposed to $\mathrm{H}_{2} \mathrm{O}_{2}$, the mRNA levels of USP14 and USP19 were increased. When myotubes were pre-incubated with $\beta$-carotene before exposure to $\mathrm{H}_{2} \mathrm{O}_{2}, \beta$-carotene dose-dependently inhibited

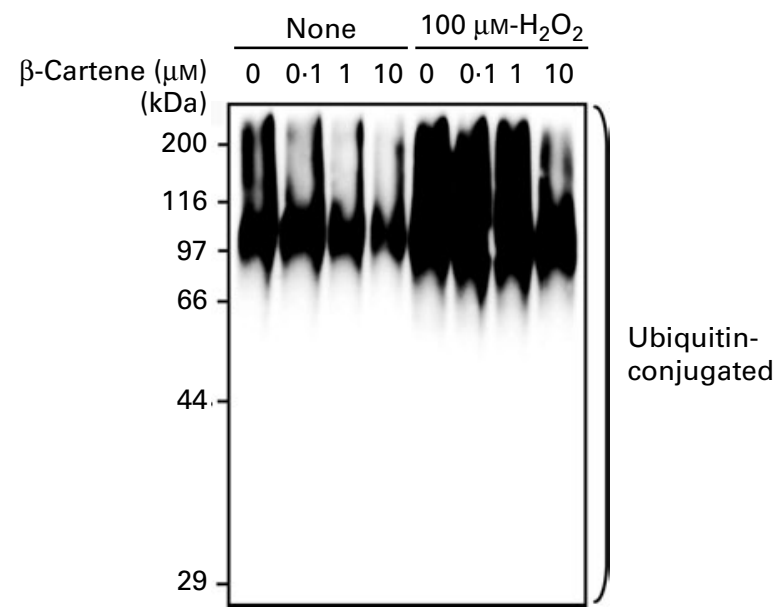

Fig. 2. Effects of $\beta$-carotene on the levels of ubiquitin conjugates in hydrogen peroxide-treated $\mathrm{C} 2 \mathrm{C} 12$ myotubes. $\mathrm{C} 2 \mathrm{C} 12$ myotubes were pre-incubated in the presence of various concentrations $(0-10 \mu \mathrm{M})$ of $\beta$-carotene for $12 \mathrm{~h}$, and further incubated in fresh medium containing $100 \mu \mathrm{M}$-hydrogen peroxide in the presence of $10 \mu \mathrm{M}-\mathrm{MG} 132$ for $12 \mathrm{~h}$. Western blot analysis with antiubiquitin antibody is shown.

$\mathrm{H}_{2} \mathrm{O}_{2}$-induced increases in USP14 and USP19 mRNA expressions (Fig. 3(C) and (D)).

\section{$\beta$-Carotene inhibits the nuclear localisation of $\mathrm{FOXO}$ a}

FOXO3a was localised throughout the cytoplasm in the presence or absence of $\beta$-carotene (Fig. 4, first and second panels from the left side). $\mathrm{H}_{2} \mathrm{O}_{2}$ treatment induced the nuclear localisation of FOXO3a (Fig. 4, third panels from left side), while $\beta$-carotene inhibited it (Fig. 4, first panels from the right side).

\section{$\beta$-Carotene inhibits the loss of soleus muscle mass induced by denervation}

To assess the effect of $\beta$-carotene on denervation-induced muscle atrophy, the experimental protocol was designed as shown in Fig. 5(A). In the soleus muscle, the ratio of muscle mass in the denervated leg to that in the sham leg was significantly higher in the $\beta$-carotene group than in the vehicle group at day 3 , but not at day 7 , after denervation (Fig. 5(B), left panel). On the contrary, there was no significant difference in the ratio of gastrocnemius muscle mass between the vehicle group and the $\beta$-carotene group (Fig. 5(B), right panel).

\section{$\beta$-Carotene represses thiobarbituric acid-reactive substances levels increased by denervation}

In the soleus muscle, denervation increased TBARS levels in the vehicle group, but not in the $\beta$-carotene group, at day 3 (Fig. 6(A)). $\beta$-Carotene reduced TBARS levels in both sham legs and denervated legs, compared with vehicle. In contrast, in the gastrocnemius muscle, denervation had no influence 
(A)

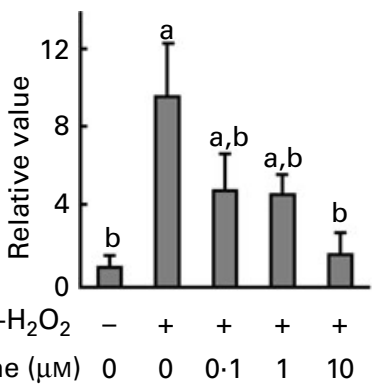

(B)

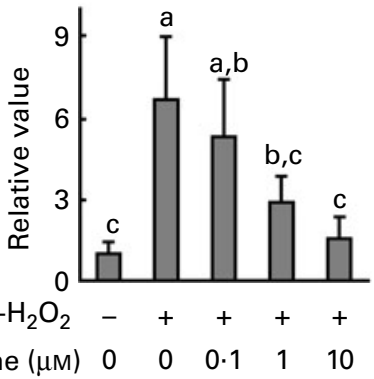

(C)

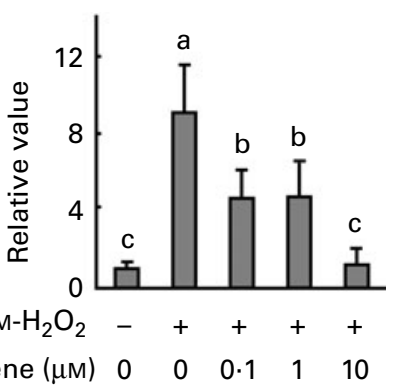

Fig. 3. Effects of $\beta$-carotene on ubiquitin ligases and deubiquitinating enzyme expressions in hydrogen peroxide-treated $\mathrm{C} 2 \mathrm{C} 12$ myotubes. $\mathrm{C} 2 \mathrm{C} 12$ myotubes were pre-incubated in the presence of various concentrations $(0-10 \mu \mathrm{M})$ of $\beta$-carotene for $12 \mathrm{~h}$, and further incubated in fresh medium containing $100 \mu \mathrm{M}$-hydrogen peroxide for $3 \mathrm{~h}$. Quantitative real-time PCR were performed for (A) Atrogin-1, (B) MuRF1, (C) USP14, (D) USP19 and GAPDH mRNA. Data were normalised to $G A P D H$ as the endogenous control. Relative values are means $(n 3)$, with their standard deviations represented by vertical bars. ${ }^{\text {,b,c }}$ Mean values with unlike letters were significantly different $(P<0.05)$.

on TBARS levels in the vehicle or $\beta$-carotene group, and $\beta$-carotene had no influence on TBARS levels in sham legs or denervated legs (Fig. 6(B)).

\section{$\beta$-Carotene inhibits the denervation-induced increased levels of ubiquitin conjugates in the soleus muscle}

We determined the effects of $\beta$-carotene on the levels of ubiquitin conjugates in soleus and gastrocnemius muscles. In soleus and gastrocnemius muscles, the levels of ubiquitin conjugates were increased by denervation (Fig. 7(A) and (B)). $\beta$-Carotene attenuated the denervation-induced accumulation of ubiquitin conjugates in the soleus muscle, but not in the gastrocnemius muscle.

\section{$\beta$-Carotene represses the expression of ubiquitin ligases and deubiquitinating enzymes increased by denervation}

Denervation significantly increased the levels of Atrogin-1, MURF1, USP14 and USP19 in the soleus muscle in the vehicle

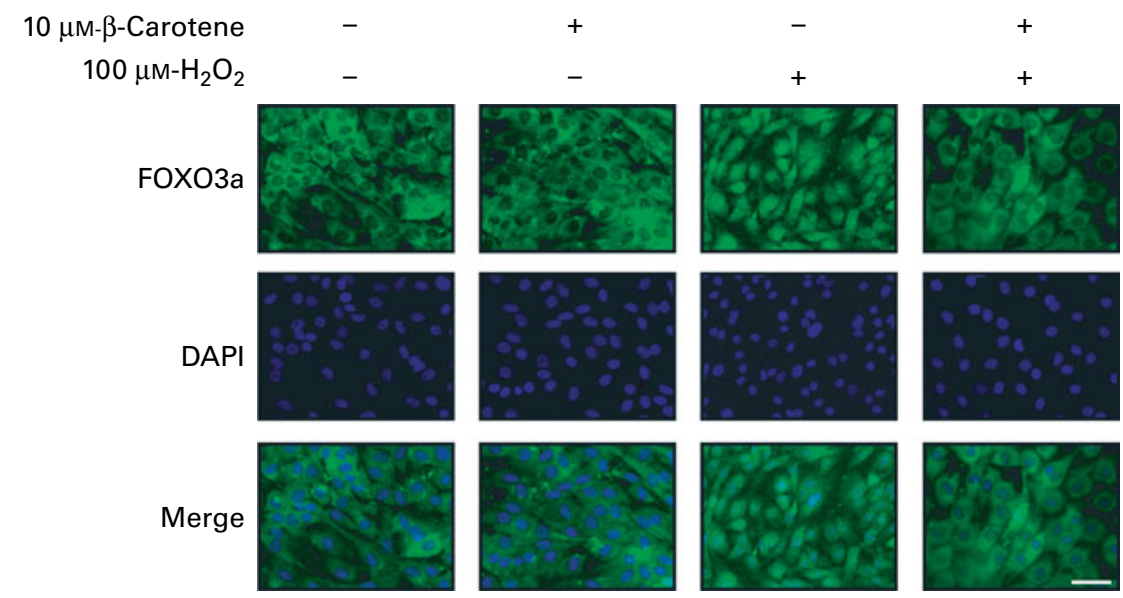

Fig. 4. Inhibitory effect of $\beta$-carotene on the nuclear localisation of $\mathrm{FOXO} 3 \mathrm{a}$ induced by hydrogen peroxide. $\mathrm{C} 2 \mathrm{C} 12$ myotubes were pre-incubated in the presence of vehicle or $10 \mu \mathrm{M}-\beta$-carotene for $12 \mathrm{~h}$, and further incubated in fresh medium containing $100 \mu \mathrm{M}$-hydrogen peroxide for $3 \mathrm{~h}$. Immunoreaction with anti-FOXO3a antibody was performed, followed by incubation with fluorescent-labelled secondary antibody. The nuclei were counterstained with 4,4',6-diamidino-2-phenylindole dihydrochloride (DAPI). The scale bar represents $100 \mu \mathrm{m}$. 
(A)

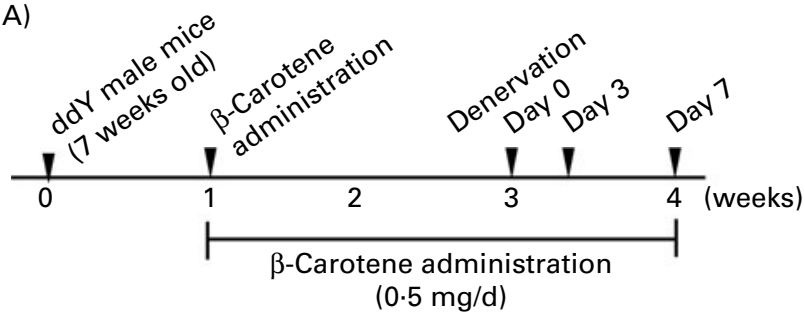

(B)
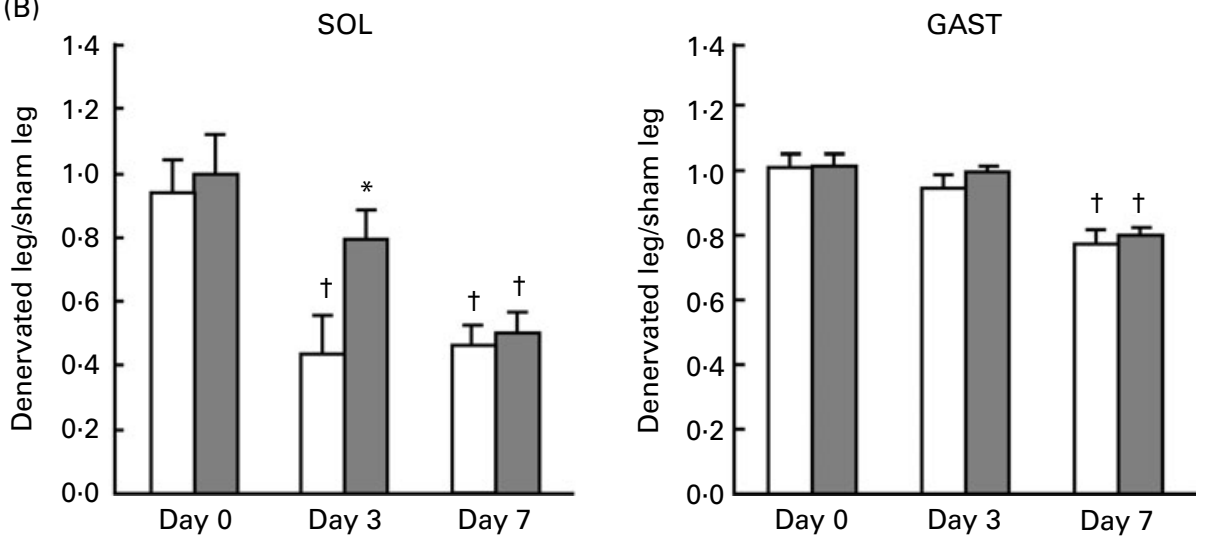

Fig. 5. Effects of $\beta$-carotene on denervation-induced skeletal muscle atrophy. (A) Experimental protocol. Micellar $\beta$-carotene was orally administered (0.5 mg once daily). After 2 weeks of administration, muscle atrophy due to denervation was induced, and once-daily administration of micellar $\beta$-carotene was performed until the end of the experiment ( $\beta$-carotene group). As a negative control, micelles without $\beta$-carotene were administered in place of micellar $\beta$-carotene (vehicle group). (B) At days 3 and 7 after denervation, the ratio of soleus muscle (SOL) or gastrocnemius muscle (GAST) mass in the denervated leg to that in the sham leg was determined. Values are means $(n 5)$, with their standard deviations represented by vertical bars. Mean value was significantly different compared with the ratio in the vehicle group at the same time point: ${ }^{*} P<0.05$. Mean value was significantly different compared with the ratio at day 0 in each group (vehicle group or $\beta$-carotene group): $\dagger P<0.05 . \square$, Vehicle; $\square, \beta$-carotene.

group at day 3 (Fig. 8). In contrast, $\beta$-carotene reduced the levels of Atrogin-1, MURF1, USP14 and USP19 in denervated legs, compared with vehicle.

\section{Discussion}

In skeletal muscle, disuse (e.g. denervation, lengthy bed rest or limb immobilisation) causes atrophy accompanied by a

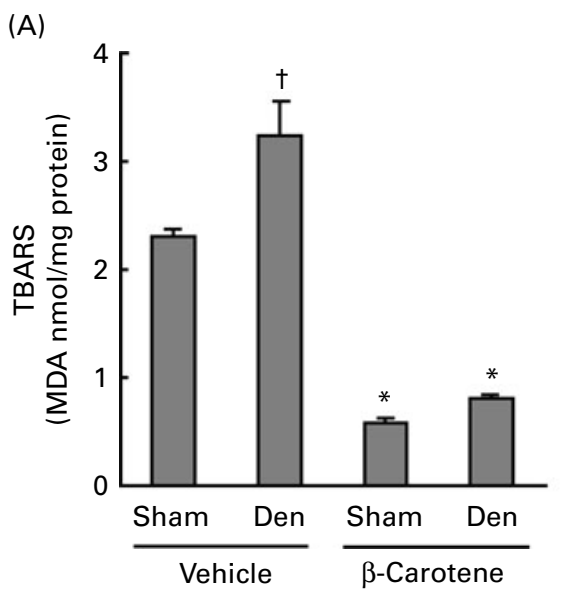

reduction of mass. Muscle atrophy results in dysfunction in muscles. Disuse causes an imbalance between reactive oxygen species production and scavenging, resulting in the accumulation of reactive oxygen species in the mitochondria $^{(28)}$. Because oxidative stress seems to be involved in disuse atrophy ${ }^{(3,4)}$, antioxidants are expected to prevent the progression of muscle atrophy and to improve the muscle mass and function. In the present study, we demonstrated

(B)

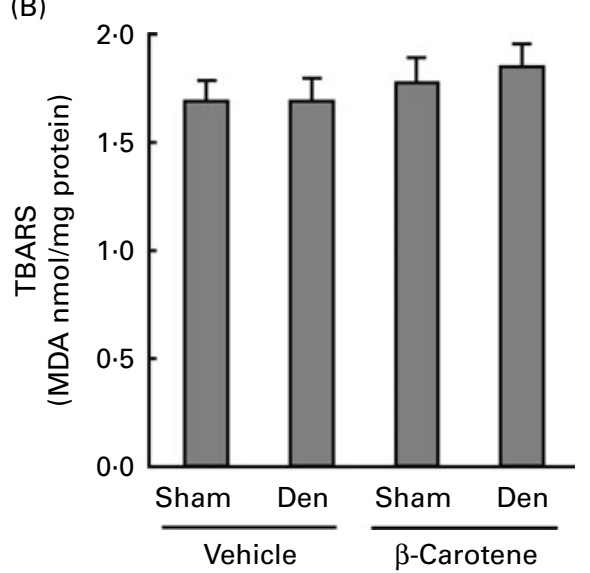

Fig. 6. Effects of $\beta$-carotene on thiobarbituric acid-reactive substances (TBARS). Mice were administrated micelles with ( $\beta$-carotene group) or without (vehicle group) $\beta$-carotene. (A) Soleus and (B) gastrocnemius muscles of sham legs (Sham) and denervated legs (Den) were homogenised, and TBARS levels were determined. Values are means (expressed in nmol malondialdehyde (MDA)/mg protein), with their standard deviations represented by vertical bars ( $n$ 4). Mean value was significantly different compared with the vehicle group in the same legs (Sham or Den): $P<0.05$. Mean value was significantly different compared with Sham in the same group (vehicle group or $\beta$-carotene group): $\uparrow P<0.05$. 
(A)

$\frac{\text { Vehicle }}{\frac{\text { Sham }}{123} \frac{\text { Den }}{123}} \frac{\text { Sham }}{123} \frac{\text { Den }}{123}$

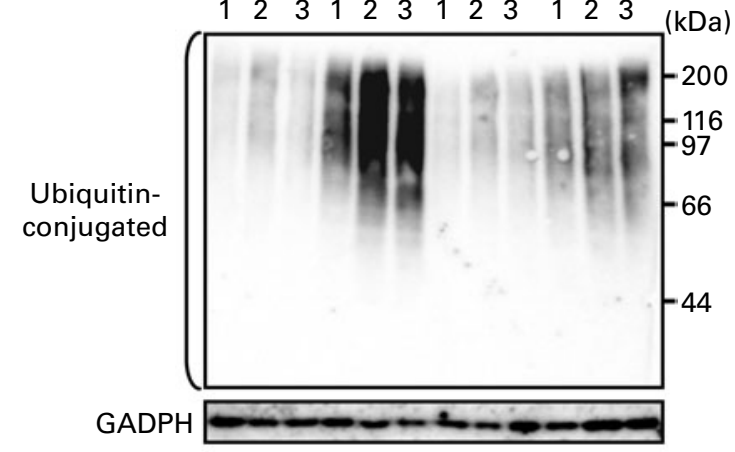

(B)

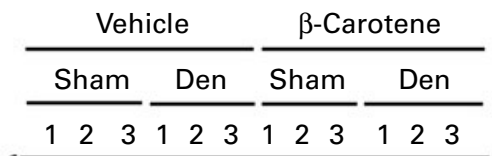

that $\beta$-carotene administration significantly prevents the loss of soleus muscle mass, but not gastrocnemius muscle mass, at day 3 after denervation. However, $\beta$-carotene did not affect the masses of soleus and gastrocnemius muscles at day 7 after denervation. Skeletal muscle fibres are classified into two major types: slow twitch and fast twitch. MyHC expresses as slow-type and fast-type isoforms, which are the major proteins responsible for each muscle fibre type. MyHC isoforms work in association with tropomyosin, which is one of the myofibrillar proteins, for muscle contraction. Thus, MyHC and tropomyosin are dominant structural proteins in differentiated muscle and are generally used as markers of myotubes. In the present study, we selected the soleus and gastrocnemius muscles as examples of slow- and fast-twitch muscles, respectively. During muscle disuse atrophy, the mass of slow-twitch soleus muscle is lost more rapidly than that of fast-twitch gastrocnemius muscle ${ }^{(7,29)}$, and protein oxidation and lipid peroxidation occur in the soleus muscle more than in the gastrocnemius muscle ${ }^{(30)}$. These results are consistent with the present results showing the decreased mass and the increased TBARS level in the soleus muscle, but not in the gastrocnemius muscle, at $3 \mathrm{~d}$ after denervation. In the present in vitro experiments, pretreatment with $\beta$-carotene inhibited the $\mathrm{H}_{2} \mathrm{O}_{2}$-induced reduction of $\mathrm{MyHC}$ and tropomyosin levels (Fig. 1). The inhibitory effects on muscle atrophy were in the order $\beta$-carotene $=\beta$-cryptoxanthin $>$ zeaxanthin, consistent with a previous report ${ }^{(31)}$ that the sequence for relative antioxidant activities is $\beta$-carotene $=\beta$-cryptoxanthin $>$ zeaxanthin. Furthermore, $\beta$-carotene inhibited the denervation-induced reduction of muscle mass and the increase in TBARS levels in the soleus muscle at day 3 . These results suggest that the antioxidant activity of $\beta$-carotene has a beneficial effect on the retardation of soleus muscle loss at the early stage of muscle atrophy (e.g. day 3).

$\beta$-Carotene administration attenuated the level of ubiquitin conjugates and the expression levels of Atrogin-1 and MURF1 induced by denervation in the soleus muscle. Vitamin E supplementation partially prevents the increases in Atrogin1 and MuRF1 mRNA levels and the decrease in soleus muscle mass induced by unloading and immobilisation ${ }^{(32,33)}$. Furthermore, $\beta$-carotene inhibited the $\mathrm{H}_{2} \mathrm{O}_{2}$-induced nuclear localisation of FOXO3a. $\mathrm{H}_{2} \mathrm{O}_{2}$ induces p38 (mitogen-activated protein kinase)- and c-Jun N-terminal kinase-mediated nuclear accumulation of FOXO3a, and nuclear FOXO3a up-regulates the expression of Atrogin-1 and $M u R F 1^{(10,13)}$. Various antioxidants inhibit the activation of p38 induced by mitochondrial oxidative damage ${ }^{(34,35)}$. These results suggest that $\beta$-carotene represses atrophy-induced Atrogin-1 and MuRF1 gene expression by inhibiting oxidative stress-activated FOXO3a signalling.

The levels of USP14 and USP19 mRNA were increased in $\mathrm{H}_{2} \mathrm{O}_{2}$-treated $\mathrm{C} 2 \mathrm{C} 12$ myotubes, and $\beta$-carotene inhibited their increases. Denervation increased the expression levels of USP14 and USP19 mRNA in the soleus muscle, whereas $\beta$-carotene significantly reduced their increased expressions. DUB also control the levels of ubiquitin conjugates in the ubiquitin-proteasome system, and the expression levels of USP14 and USP19 mRNA are increased during muscle atrophy $^{(15,16)}$. Although the levels of Atrogin-1 and MuRF1 are usually measured during muscle atrophy, the levels of DUB such as USP14 and USP19 also should be monitored as atrogenes.

The beneficial effects of $\beta$-carotene on muscle atrophy were lost on day 7 (Fig. 5(B)). The expressions of Atrogin-1 and MuRF1 reach a peak at day 3 (during the early phase) after disuse atrophy due to denervation and then return to basal levels at day 14 after denervation ${ }^{(8)}$. However, the relative muscle weight to body weight continues to decrease even after $14 \mathrm{~d}$ post-denervation. These results suggest that the early and later phases of muscle atrophy due to denervation are regulated by at least two distinct mechanisms. $\beta$-Carotene seems to have a beneficial effect on muscle atrophy induced by the ubiquitin-proteasome system during the early phase of muscle atrophy (e.g. day 3).

We demonstrated the beneficial effects of $\beta$-carotene on $\mathrm{H}_{2} \mathrm{O}_{2}$-induced muscle atrophy in $\mathrm{C} 2 \mathrm{C} 12$ myotubes. When human subjects receive daily administration of $\beta$-carotene 

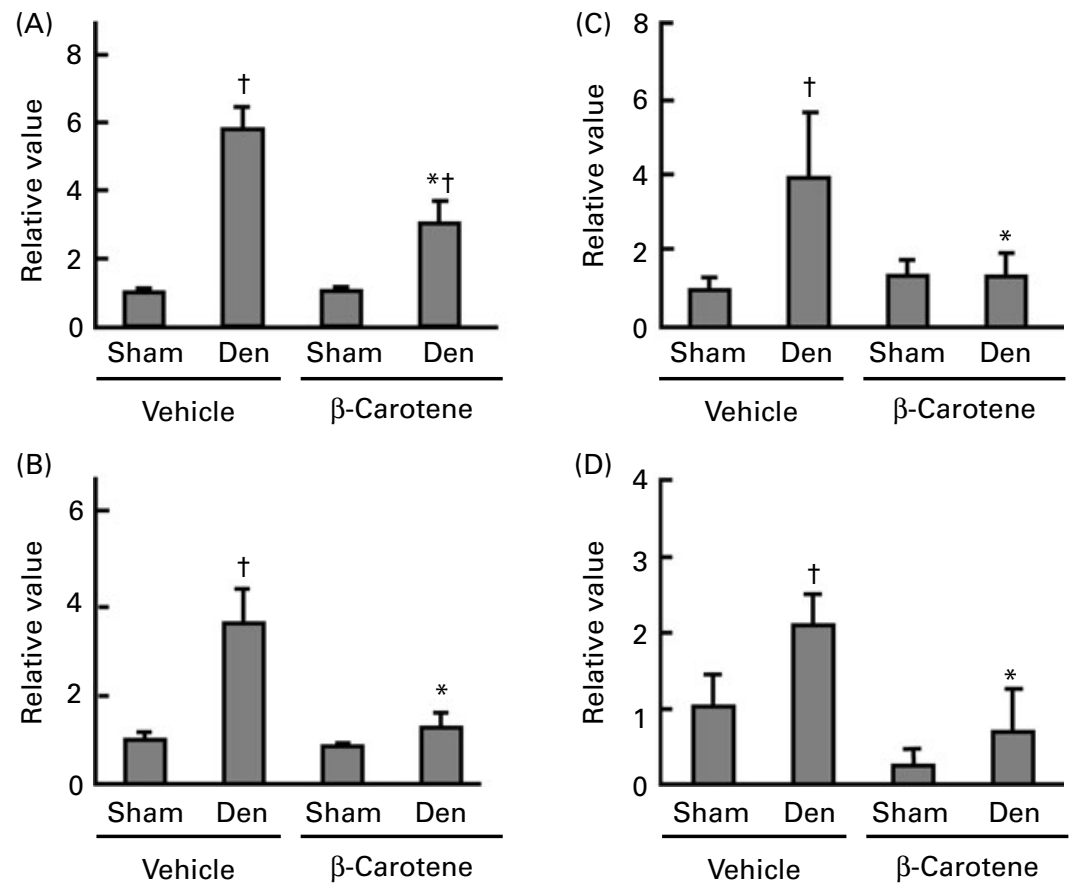

Fig. 8. Effects of $\beta$-carotene on ubiquitin ligase and deubiquitinating enzyme expressions in the denervated soleus muscle. Mice were administrated micelles with ( $\beta$-carotene group) or without (vehicle group) $\beta$-carotene. Total RNA was prepared from the soleus muscle in denervated legs (Den) and sham legs (Sham) at day 3 after denervation. (A) Atrogin-1, (B) MuRF1, (C) USP14, (D) USP19 and $\beta$-actin mRNA levels were analysed by quantitative real-time PCR. Data were normalised to $\beta$-actin. Values are means $(n 5)$, with their standard deviations represented by vertical bars. Mean value was significantly different compared with the vehicle group in the legs with the same treatment (Sham or Den): ${ }^{*} P<0.05$. Mean value was significantly different compared with Sham in the same group (vehicle group or $\beta$-carotene group): $† P<0.05$.

( $45 \mathrm{mg}$ ) for $30 \mathrm{~d}$, plasma concentrations of $\beta$-carotene are increased within $2-4 \mathrm{~d}$ after administration and then remain elevated $^{(36)}$. Although there are inter-individual differences in the extent of the increase in $\beta$-carotene concentrations in plasma, $\beta$-carotene concentrations are increased to approximately $5 \mu \mathrm{M}$ in some subjects. On the other hand, plasma concentrations of $\beta$-carotene after $\beta$-carotene supplementation $(20 \mathrm{mg} / \mathrm{d})$ for 3 years reach up to $5.6 \mu \mathrm{m}$ in human subjects ${ }^{(37)}$. Therefore, we investigated the effects of $\beta$-carotene at physiologically achievable concentrations $(0 \cdot 1-10 \mu \mathrm{M})$ on oxidative stress-induced muscle atrophy in $\mathrm{C} 2 \mathrm{C} 12$ myotubes. Although most of the carrots (Daucus carota L.) contain $\beta$-carotene at $>10 \mathrm{mg} / 100 \mathrm{~g}$ dry weight ${ }^{(38,39)}$, humans would need to take $\beta$-carotene as a supplement rather than as a regular diet to increase plasma $\beta$-carotene concentrations to $5-10 \mu \mathrm{m}$.

Absorption efficiency of carotenoids in rodents is generally poorer than that in humans ${ }^{(40)}$. However, absorption efficiency of $\beta$-carotene is enhanced in rodents when $\beta$-carotene is solubilised in phospholipid-mixed micelles ${ }^{(41)}$. In OUTBWistar rats ( $42 \mathrm{~g}$ body weight), the plasma concentration of $\beta$-carotene is approximately $0 \cdot 2 \mu \mathrm{m}$ at $1 \mathrm{~h}$ after oral administration of $\beta$-carotene (approximately $20 \mu \mathrm{g}$ ) in micelles. These results suggest that approximately $15 \mu \mathrm{g} \beta$-carotene in micelles is required for ddY mice ( $30-35 \mathrm{~g}$ body weight) to increase plasma $\beta$-carotene concentrations to $0.2 \mu \mathrm{m}$. Furthermore, because absorption of $\beta$-carotene is increased in a dose-dependent manner ${ }^{(42)}$, we predicted that administration of a 25 - to 50 -fold excess of $\beta$-carotene $(0.375-0.750 \mathrm{mg})$ would be required to increase plasma $\beta$-carotene concentration to $5-10 \mu \mathrm{m}$. We are attempting to determine the pharmacokinetic properties of $\beta$-carotene in plasma in mice administered $0.5 \mathrm{mg} \beta$-carotene.

We have demonstrated that dietary supplementation of $\beta$-carotene is useful as a chemopreventive agent for improvement of disuse-induced muscle mass loss at the early stage of soleus muscle atrophy. Because decreasing the nuclear FOXO3a expression level is effective in repressing the progression of muscle atrophy, it is of interest to find dietary antioxidants that prevent oxidative stress-induced nuclear localisation of FOXO3a. Furthermore, the mechanism by which the expression of DUB is induced by oxidative stress remains to be elucidated.

\section{Acknowledgements}

This study was supported by a Grant-in-Aid (23580182) for scientific research (to R. Y.) from the Japan Society for the Promotion of Science. We are grateful to Mana Ishikawa for helpful support in the animal experiments. M. O. contributed to the data analysis and manuscript preparation. Y. K. and T. K. analysed the data. R. Y. drafted the manuscript and contributed to the study design. N. H. and T. S. conducted the data analysis. K. H., Y. N. and H. I. were involved in the discussion and design of the study. The authors declare that there is no conflict of interest. 


\section{References}

1. Powers SK, Kavazis AN \& McClung JM (2007) Oxidative stress and disuse muscle atrophy. J Appl Physiol 102, 2389-2397.

2. Furuno K, Goodman MN \& Goldberg AL (1990) Role of different proteolytic systems in the degradation of muscle proteins during denervation atrophy. $J$ Biol Chem 265, $8850-8857$.

3. Lawler JM, Song W \& Demaree SR (2003) Hindlimb unloading increases oxidative stress and disrupts antioxidant capacity in skeletal muscle. Free Radic Biol Med 35, 9-16.

4. Servais S, Letexier D, Favier R, et al. (2007) Prevention of unloading-induced atrophy by vitamin $\mathrm{E}$ supplementation: links between oxidative stress and soleus muscle proteolysis? Free Radic Biol Med 42, 627-635.

5. Mukai R, Nakao R, Yamamoto H, et al. (2010) Quercetin prevents unloading-derived disused muscle atrophy by attenuating the induction of ubiquitin ligases in tail-suspension mice. J Nat Prod 73, 1708-1710.

6. Bodine SC, Latres E, Baumhueter S, et al. (2001) Identification of ubiquitin ligases required for skeletal muscle atrophy. Science 294, 1704-1708.

7. Okamoto T, Torii S \& Machida S (2011) Differential gene expression of muscle-specific ubiquitin ligase MAFbx/Atrogin-1 and MuRF1 in response to immobilization-induced atrophy of slow-twitch and fast-twitch muscles. J Physiol Sci 61, 537-546.

8. Sacheck JM, Hyatt JP, Raffaello A, et al. (2007) Rapid disuse and denervation atrophy involve transcriptional changes similar to those of muscle wasting during systemic diseases. FASEB J 21, 140-155.

9. Jagoe RT, Lecker SH, Gomes M, et al. (2002) Patterns of gene expression in atrophying skeletal muscles: response to food deprivation. FASEB J 16, 1697-1712.

10. Sandri M, Sandri C, Gilbert A, et al. (2004) Foxo transcription factors induce the atrophy-related ubiquitin ligase atrogin-1 and cause skeletal muscle atrophy. Cell 117, 399-412.

11. Li YP, Lecker SH, Chen Y, et al. (2003) TNF-alpha increases ubiquitin-conjugating activity in skeletal muscle by up-regulating UbcH2/E220k. FASEB J 17, 1048-1057.

12. Brunet A, Sweeney LB, Sturgill JF, et al. (2004) Stress-dependent regulation of FOXO transcription factors by the SIRT1 deacetylase. Science 303, 2011-2015.

13. Clavel S, Siffroi-Fernandez S, Coldefy AS, et al. (2010) Regulation of the intracellular localization of Foxo3a by stressactivated protein kinase signaling pathways in skeletal muscle cells. Mol Cell Biol 30, 470-480.

14. Komander D, Clague MJ \& Urbé S (2009) Breaking the chains: structure and function of the deubiquitinases. Nat Rev Mol Cell Biol 10, 550-563.

15. Lecker SH, Jagoe RT, Gilbert A, et al. (2004) Multiple types of skeletal muscle atrophy involve a common program of changes in gene expression. FASEB J 18, 39-51.

16. Combaret L, Adegoke OA, Bedard N, et al. (2005) USP19 is a ubiquitin-specific protease regulated in rat skeletal muscle during catabolic states. Am J Physiol Endocrinol Metab 288, E693-E700.

17. Sundaram P, Pang Z, Miao M, et al. (2009) USP19-deubiquitinating enzyme regulates levels of major myofibrillar proteins in L6 muscle cells. Am J Physiol Endocrinol Metab 297, E1283-E1290.

18. Sies H \& Stahl W (1995) Vitamins E and C, $\beta$-carotene, and other carotenoids as antioxidants. Am J Clin Nutr 62, 1315S-1321S.
19. Paiva SA \& Russell RM (1999) $\beta$-Carotene and other carotenoids as antioxidants. J Am Coll Nutr 18, 426-433.

20. Kameji H, Mochizuki K, Miyoshi N, et al. (2010) $\beta$-Carotene accumulation in 3T3-L1 adipocytes inhibits the elevation of reactive oxygen species and the suppression of genes related to insulin sensitivity induced by tumor necrosis factor- $\alpha$. Nutrition 26, 1151-1156.

21. Palozza P, Serini S, Di Nicuolo F, et al. (2003) Prooxidant effects of beta-carotene in cultured cells. Mol Aspects Med 24, 353-362.

22. Kim Y, Seo JH \& Kim H (2011) $\beta$-Carotene and lutein inhibit hydrogen peroxide-induced activation of NF- $\mathrm{BB}$ and IL-8 expression in gastric epithelial AGS cells. J Nutr Sci Vitaminol (Tokyo) 57, 216-223.

23. Hashimoto T, Ozaki Y, Taminato M, et al. (2009) The distribution and accumulation of fucoxanthin and its metabolites after oral administration in mice. Br J Nutr 102, $242-248$.

24. Ogawa M, Yamaji R, Higashimura Y, et al. (2011) 17ß-Estradiol represses myogenic differentiation by increasing ubiquitin-specific peptidase 19 through estrogen receptor $\alpha$. J Biol Chem 286, 41455-41465.

25. Yamaji R, Fujita K, Takahashi S, et al. (2003) Hypoxia up-regulates glyceraldehyde-3-phosphate dehydrogenase in mouse brain capillary endothelial cells: involvement of $\mathrm{Na}^{+} / \mathrm{Ca}^{2+}$ exchanger. Biochim Biophys Acta 1593, 269-276.

26. Yamaji R, Chatani E, Harada N, et al. (2005) Glyceraldehyde3-phosphate dehydrogenase in the extracellular space inhibits cell spreading. Biochim Biophys Acta 1726, 261-271.

27. Ohkawa H, Ohishi N \& Yagi K (1979) Assay for lipid peroxides in animal tissues by thiobarbituric acid reaction. Anal Biochem 95, 351-358.

28. Muller FL, Song W, Jang YC, et al. (2007) Denervationinduced skeletal muscle atrophy is associated with increased mitochondrial ROS production. Am J Physiol Regul Integr Comp Physiol 293, R1159-R1168.

29. Dehority W, Halloran BP, Bikle DD, et al. (1999) Bone and hormonal changes induced by skeletal unloading in the mature male rat. Am J Physiol 276, E62-E69.

30. Brocca L, Pellegrino MA, Desaphy JF, et al. (2010) Is oxidative stress a cause or consequence of disuse muscle atrophy in mice? A proteomic approach in hindlimb-unloaded mice. Exp Physiol 95, 331-350.

31. Miller NJ, Sampson J, Candeias LP, et al. (1996) Antioxidant activities of carotenes and xanthophylls. FEBS Lett $\mathbf{3 8 4}$, $240-242$

32. Appell HJ, Duarte JA \& Soares JM (1997) Supplementation of vitamin E may attenuate skeletal muscle immobilization atrophy. Int J Sports Med 18, 157-160.

33. Kondo H, Miura M, Nakagaki I, et al. (1992) Trace element movement and oxidative stress in skeletal muscle atrophied by immobilization. Am J Physiol 262, E583-E590.

34. Peus D, Vasa RA, Beyerle A, et al. (1999) UVB activates ERK1/2 and p38 signaling pathways via reactive oxygen species in cultured keratinocytes. J Invest Dermatol 112, 751-756

35. Gaitanaki C, Papatriantafyllou M, Stathopoulou $\mathrm{K}$, et al. (2006) Effects of various oxidants and antioxidants on the p38-MAPK signalling pathway in the perfused amphibian heart. Mol Cell Biochem 291, 107-117.

36. Dimitrov NV, Meyer C, Ullrey DE, et al. (1988) Bioavailability of beta-carotene in humans. Am J Clin Nutr 48, 298-304. 
37. Albanes D \& Heinonen (1994) The effect of vitamin E and beta carotene on the incidence of lung cancer and other cancers in male smokers. N Engl J Med 330, 1029-1035.

38. Mech-Nowak A, Swiderski A, Kruczek M, et al. (2012) Content of carotenoids in roots of seventeen cultivars of Daucus carota L. Acta Biochim Pol 59, 139-141.

39. Surles RL, Weng N, Simon PW, et al. (2004) Carotenoid profiles and consumer sensory evaluation of specialty carrots (Daucus carota L.) of various colors. J Agric Food Chem $\mathbf{5 2}$ 3417-3421.
40. Moon RC \& Constantinou AI (1997) Dietary retinoids and carotenoids in rodent models of mammary tumorigenesis. Breast Cancer Res Treat 46, 181-189.

41. Marisiddaiah R \& Baskaran V (2009) Bioefficacy of beta-carotene is improved in rats after solubilized as equimolar dose of beta-carotene and lutein in phospholipid-mixed micelles. Nutr Res 29, 588-595.

42. Hollander D \& Ruble PE Jr (1978) Beta-carotene intestinal absorption: bile, fatty acid, $\mathrm{pH}$, and flow rate effects on transport. Am J Physiol 235, E686-E691. 